\title{
Western Blot in Maize
}

María Jazmín Abraham-Juárez*

CONACYT. Molecular Biology Department, Instituto Potosino de Investigación Científica y Tecnológica (IPICYT), San Luis Potosí, SLP, Mexico

*For correspondence: maria.abraham@ipicyt.edu.mx

[Abstract] Since its introduction in 1979 (Towbin et al., 1979), protein blotting (Western blot) has become a routine tool in molecular biology research. It is used to detect low amounts of proteins in complex samples or to monitor protein expression and purification. Western blotting includes protein complex separation by gel electrophoresis (SDS-PAGE), transfer to a fixed matrix or membrane and analysis by immunodetection using a specific antibody against the protein of interest. This method provides molecular mass information of individual proteins, and it can be used to distinguish between isoforms and post-translational modification states. There are many variations on the Western blot procedure that can be performed to improve detection, specificity, and quantitative power. In plants, Western blot has to be standardized to get a satisfactory signal and reproducible results, due to the complex nature of cell extracts. Here, I describe a protocol for a semi-quantitative Western blot using total maize protein and specific polyclonal antibodies raised against maize proteins of interest. The data are analyzed with ImageJ.

Keywords: Maize, Protein, Western blot, ImageJ

\section{Materials and Reagents}

1. Protease-free pipette tips $(1,000,200$ and $10 \mu l)$

2. $1.5 \mathrm{ml}$ Eppendorf tubes

3. Immuno-Blot PVDF Membrane, Roll, $26 \mathrm{~cm} \times 3.3 \mathrm{~m}$ (Bio-Rad, catalog number: 1620177)

4. Filter paper squares, $7.5 \mathrm{~cm} \times 8.4 \mathrm{~cm}$

5. $12 \times 12 \mathrm{~cm}$ Square Petri dishes

6. Transparent plastic wrap

7. X-ray films

8. Paper disposable wipes

9. Tris base (Sigma, catalog number: T1503)

10. Sodium chloride (Sigma, catalog number: S7653)

11. IGEPAL-CA-630 (Sigma, catalog number: I8896)

12. Complete ${ }^{\mathrm{TM}}$ Protease Inhibitor Cocktail tablets (Sigma, catalog number: 11697498001)

13. PhosSTOP'M Phosphatase inhibitor tablets (Sigma, catalog number: 4906845001)

14. DL Dithiothreitol (Sigma, catalog number: 43819)

15. Sucrose for molecular biology (Sigma, catalog number: S0389) 
16. Glycerol for molecular biology (Sigma, catalog number: G5516)

17. Methanol for HPLC (Sigma, catalog number: 34860)

18. Ethanol anhydrous (Sigma, catalog number: 459836)

19. Tween 20 for molecular biology (Sigma, catalog number: P9416)

20. 30\% Acrylamide/Bis Solution, 29:1 (Bio-Rad, catalog number: 1610156)

21. EDTA, Disodium Salt, Dihydrate (Sigma, catalog number: 324503)

22. Magnesium chloride $\left(\mathrm{MgCl}_{2}\right)$ (Sigma, catalog number: M8266)

23. $\beta$-mercaptoethanol (Sigma, catalog number: M6250)

24. Sodium dodecyl sulfate, SDS (Sigma, catalog number: 436143)

25. Bromophenol blue (Bio-Rad, catalog number: 1610404)

26. Ammonium persulfate for electrophoresis, $\geq 98 \%$ (Sigma, catalog number: A3678)

27. Prestained protein ladder, PageRuler 10 to 180 kDa (Thermo Fisher Scientific, catalog number: 26616)

28. Nonfat-Dried Milk (Sigma, catalog number: M7409-1BTL)

29. Bovine serum albumin (Sigma, catalog number: A3311)

30. Primary antibody Anti-NOD (Rosa et al., 2017)

31. Secondary antibody Polyclonal Anti-Guinea pig HRP-conjugated produced in Goat (Thermo Fisher Scientific, catalog number: A18769)

32. Restore Western blot stripping buffer (Thermo Fisher Scientific, catalog number: 21059)

33. Clarity Western ECL Substrate (Bio-Rad, catalog number: 1705061) (see Note 2)

34. Clarity Max Western ECL Substrate (Bio-Rad, catalog number: 1705062S) (see Note 2)

35. NBT/BCIP substrate ready to use tablets (Sigma, catalog number: 11697471001)

36. Glycine for electrophoresis (Sigma, catalog number: G8898)

37. Bovine serum albumin (BSA) (Sigma, catalog number: A9647)

38. Tris base (Sigma, catalog number: T1503)

39. TEMED $\left(N, N, N^{\prime}, N^{\prime}-\right.$ Tetramethylethylenediamine) for electrophoresis (Sigma, catalog number: T9281)

40. MQ water and distilled water

41. Total protein extraction buffer (see Recipes)

42. $5 x$ Loading buffer (see Recipes)

43. $1 x$ Tris-Glycine running buffer (see Recipes)

44. Transfer buffer (see Recipes)

45. 1x TBST buffer (see Recipes)

46. Ammonium persulfate (APS) (see Recipes)

47. Blocking solution (see Recipes)

48. Blocking solution for detection of phosphorylated proteins (see Recipes)

49. Ponceau S Staining Solution (see Recipes) 


\section{Equipment}

1. Pipettes

2. Benchtop centrifuge

3. Vortex homogenizer

4. Thermoblock

5. Equipment for SDS-PAGE and Western blot

Note: For SDS-PAGE, we have had success with the Bio-Rad electrophoresis equipment. For transfer, a semi dry Blotter is very good when working with proteins from 10 to $120 \mathrm{kDa}$ because only 10 to $20 \mathrm{~min}$ at $15 \mathrm{~V}$ at room temperature is enough for complete transfer. When working with proteins with higher molecular weight, it is better to use wet transfer, it will take $1 \mathrm{~h}$ at $94 \mathrm{~V}$ on ice bucket for complete transfer.

6. Forceps

7. Platform rocker

8. Trans-blot Turbo Transfer (Bio-Rad) or Fast Semi Dry Blotter (Thermo Scientific)

9. X-ray film cassette and X-ray film developer machine or a Chemi-doc imager machine

10. Office scanner

\section{Software}

1. ImageJ (https://imagej.nih.gov/ij/)

\section{Procedure}

1. Prepare a $12 \%$ polyacrylamide gel for SDS-PAGE, by mixing the reagents in Table 1 , in the specified order.

Table 1. $12 \%$ polyacrylamide gel components

\begin{tabular}{|c|c|}
\hline Resolving (12\%) & Stacking (4\%) \\
\hline MQ water $1.6 \mathrm{ml}$ & MQ water $1.44 \mathrm{ml}$ \\
\hline $30 \%$ Acrylamide $/$ Bis $2.0 \mathrm{ml}$ & $30 \%$ Acrylamide $/$ Bis $0.27 \mathrm{ml}$ \\
\hline $1.5 \mathrm{M}$ Tris $\mathrm{HCl}$ pH $8.81 .3 \mathrm{ml}$ & $0.5 \mathrm{M}$ Tris $\mathrm{HCl} \mathrm{pH} 6.80 .25 \mathrm{ml}$ \\
\hline $10 \%$ SDS $50 \mu \mathrm{l}$ & $10 \%$ SDS $20 \mu \mathrm{l}$ \\
\hline $10 \%$ APS $50 \mu \mathrm{l}$ & $10 \%$ APS $20 \mu \mathrm{l}$ \\
\hline TEMED $6 \mu \mathrm{l}$ & TEMED $5 \mu \mathrm{l}$ \\
\hline
\end{tabular}


a. Use a $50 \mathrm{ml}$ Falcon tube for preparation of each mix (resolving and stacking gel mixes). Addition of TEMED initiates polymerization, so do not add this until you are ready to pour the gel because after it is added you need to pour it within $2 \mathrm{~min}$.

Note: Prepare gel for SDS-PAGE a day before use it and store it at $4{ }^{\circ} \mathrm{C}$, to let the acrylamide completely polymerize. Add TEMED to the mix in a fume hood, it is very toxic.

b. Test the gel cast sealing by adding $1 \mathrm{ml}$ of ethanol anhydrous into the glass sandwich and label the level with a marker pen. If after 5 min no leaks are visible it is ready to use. Add TEMED to the resolving gel, mix completely and quickly pour $4.5 \mathrm{ml}$ into the glass sandwich (Figure 1A).

c. Add $1 \mathrm{ml}$ of ethanol anhydrous to the gel (this is to set up a smooth surface) and incubate $15 \mathrm{~min}$ at room temperature for polymerization.

d. Pour off the ethanol to a paper towel. Add TEMED to the stacking gel, mix completely and pour $1.5 \mathrm{ml}$ on top of the resolving gel. Quickly insert the comb into the liquid stacking gel. Try to avoid getting bubbles in the wells (Figure 1B).

e. Incubate $10 \mathrm{~min}$ at room temperature for polymerization. Store the gel in a plastic wrap at 4 ${ }^{\circ} \mathrm{C}$ overnight for complete polymerization.

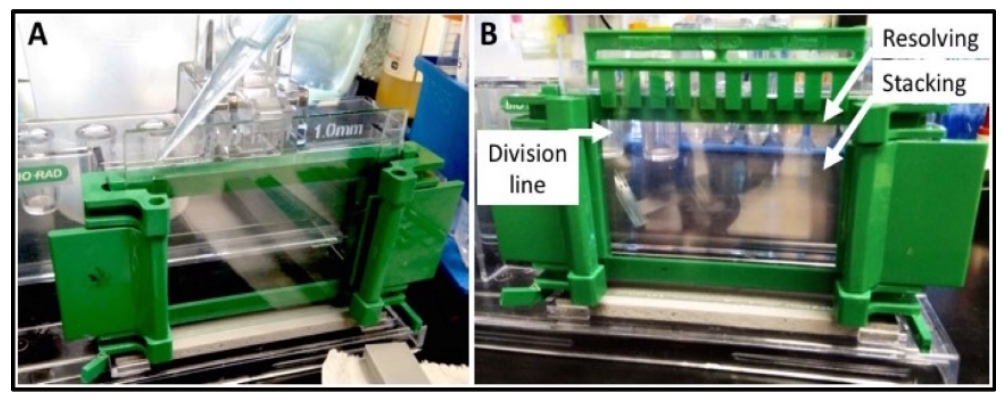

Figure 1. Polyacrylamide gel preparation. A. pouring the stacking gel. B. Gel after the comb is set.

2. Make a protein extract from maize tissue as described in Abraham-Juárez, 2019. The extraction buffer in this protocol (see Recipes) is useful for most maize proteins. For western blot, use $100 \mathrm{mg}$ of tissue and add $200 \mu \mathrm{l}$ of extraction buffer. Adjust all the protein samples to the same concentration (usually around $2 \mathrm{mg} / \mathrm{ml}$ of protein is a good concentration). Twenty to thirty micrograms per lane is usually enough for Western blot, so the volume ( 10 and $15 \mu l)$ fits within the well.

3. Mix samples with $5 x$ loading buffer at $1 x$ final concentration (add $2.5 \mu \mathrm{l}$ of loading buffer to $10 \mu \mathrm{l}$ of sample) and boil at $95{ }^{\circ} \mathrm{C}$ for $5 \mathrm{~min}$. Spin the samples for $30 \mathrm{~s}$ at room temperature at $15,000 \times g$ to collect the sample to the bottom of the tube.

4. Load the samples in a $12 \%$ polyacrylamide gel (Video 1 ). Load $5 \mu$ of prestained protein ladder (the prestained ladder will be used to estimate the size of the bands in the membrane). 


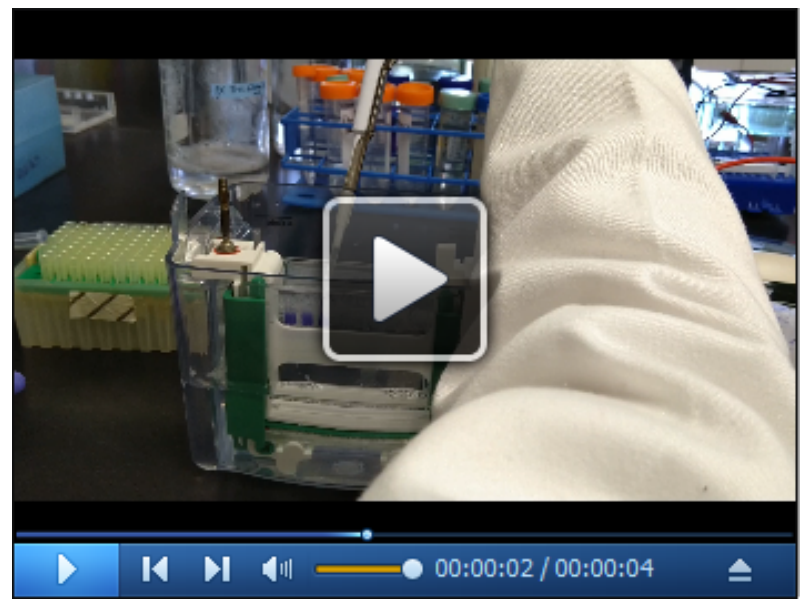

Video 1. Protein sample loading in a polyacrylamide gel

5. Run the gel with a constant voltage at $80 \mathrm{~V}$ until the dye front is at the end of the stacking gel (Figure $2 \mathrm{~A}$ ), then increase to voltage to $120 \mathrm{~V}$ until the dye front reaches the bottom of the gel (Figure 2B).

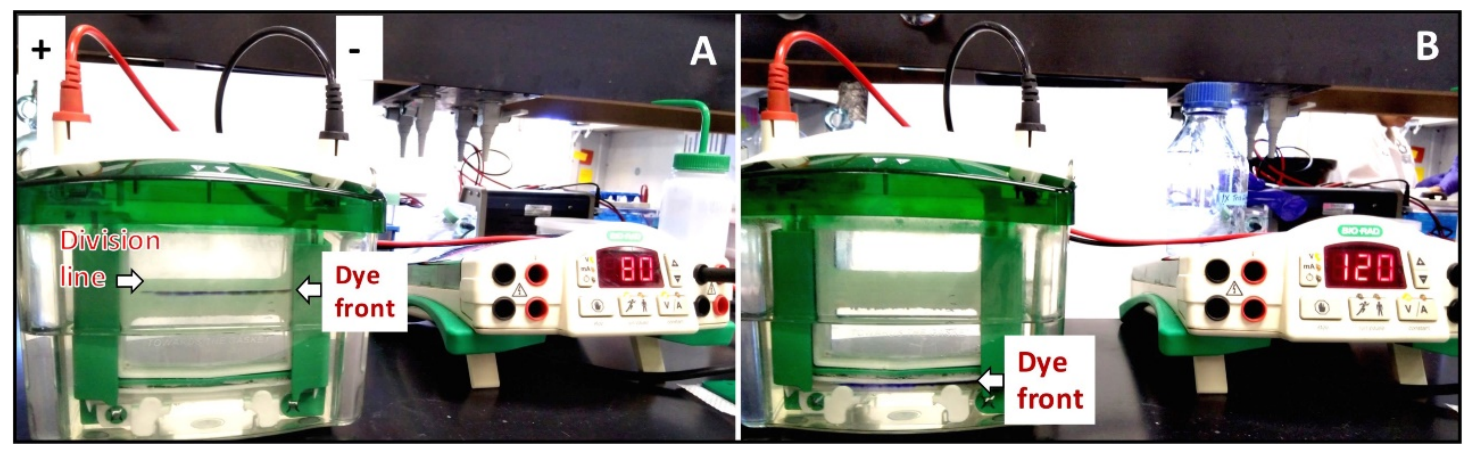

Figure 2. SDS-PAGE electrophoresis system. A. Anode (+) and Cathode (-) are shown. Arrows show the stacking-resolving division line and the dye front. B. Dye front when the run is finished.

6. After SDS-PAGE, remove the gel from glass plates and discard the stacking gel (Video 2). Equilibrate the resolving gel in $100 \mathrm{ml}$ of transfer buffer for $15 \mathrm{~min}$, in a square box with lid. 


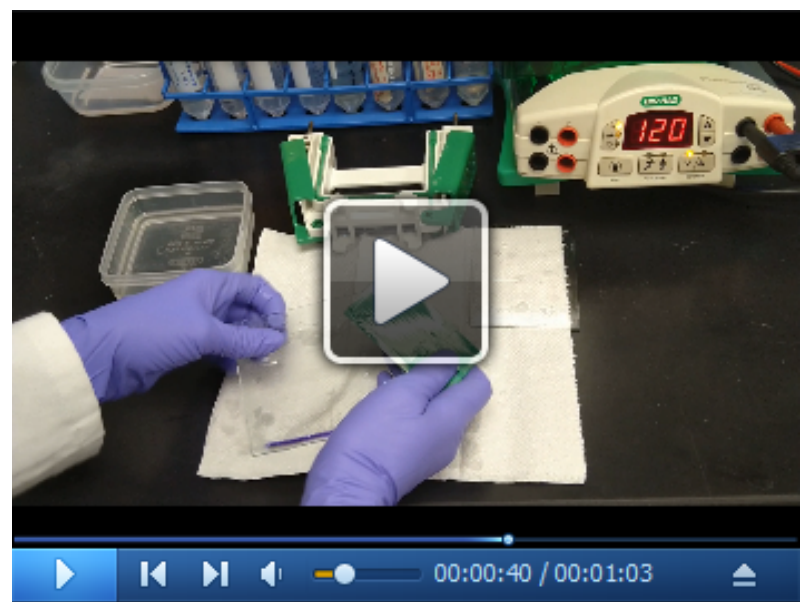

Video 2. Disassembly and gel removal

7. Cut a PVDF membrane that is the same size as the gel and soak it in $10 \mathrm{ml}$ of $100 \%$ methanol in a square Petri dish for $1 \mathrm{~min}$.

8. Use forceps to transfer the soaked membrane to the box containing the gel with transfer buffer. Equilibrate it for $5 \mathrm{~min}$.

9. Wet two thick Blotting Filter Paper squares or two sets of 3 thin filter paper squares in the same transfer buffer containing the gel and the membrane.

10. Assemble a sandwich as shown in Figure $3 A$ (Video 3 ) for transfer of protein to the membrane. Use forceps to place a soaked 3-layer filter paper on the bottom side of the semi dry blotter then place the equilibrated PVDF membrane over the filter paper. Place the gel over the membrane, trying to line it up very well. Then place another soaked 3-layer filter paper. After each layer of the transfer pack (sandwich), gently remove all the air bubbles using a roller. Finally, close the blotter firmly. 


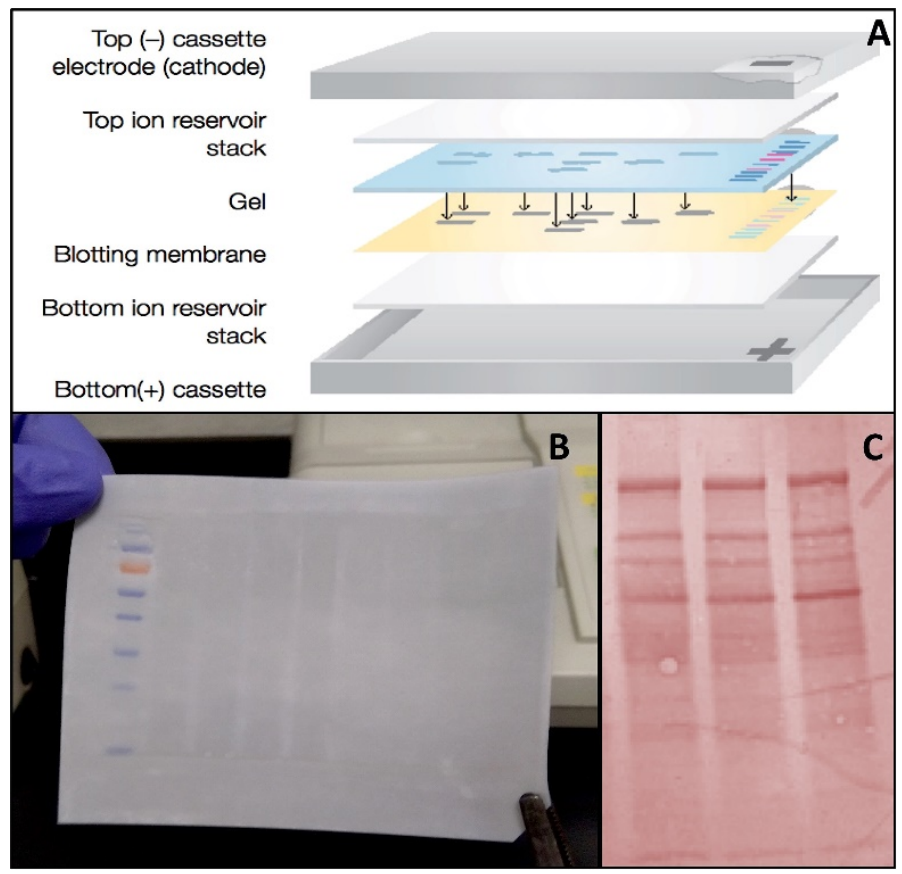

Figure 3. Blotting transfer. A. Diagram of proper layering of the transfer pack (BioRad Transblot Turbo Transfer $\left.{ }^{\circledR}\right)$. The order is from Anode (+) to Cathode (-). Bottom ion reservoir stack is the soaked 3-layer filter paper. B. Membrane after transfer, before drying. C. Ponceau S staining.

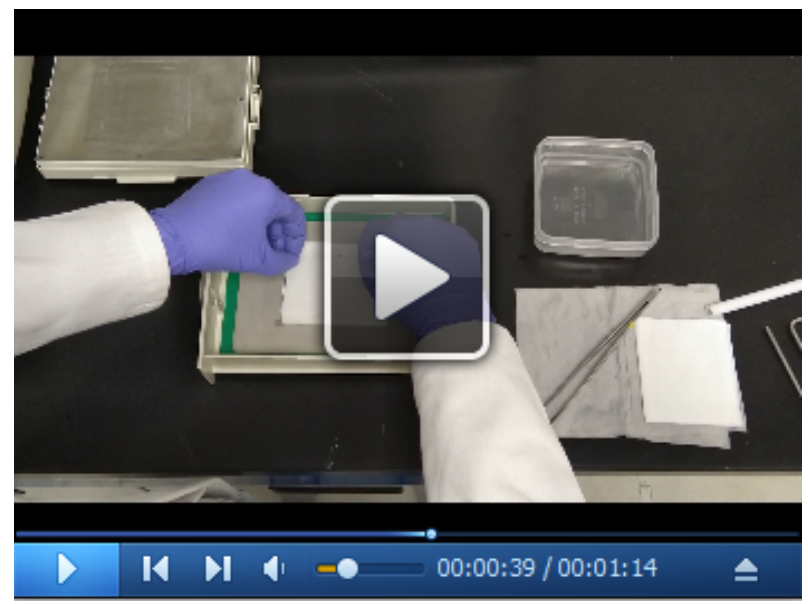

Video 3. Assembly of the transfer pack in a semi-dry blotter

11. Transfer for $20 \mathrm{~min}$ at $15 \mathrm{~V}$ in the semidry blotter, or follow the specifications of your particular blotter for 1 mini gel. When done, open the blotter, remove the filter papers and the gel. Take the membrane with forceps (Figure 3B) and lay it over two soft paper layers (paper disposable wipes) to dry it for 15 to $30 \mathrm{~min}$ at room temperature.

12. Use forceps to pick up the membrane and soak the dry membrane in 100\% methanol for $1 \mathrm{~min}$ and then in $1 \times$ TBST for 5 min with gentle shaking. A $12 \times 12 \mathrm{~cm}$ square Petri dish will be used for all the membrane washes and incubations. 
13. If confirmation of protein transfer is desired, then you can stain with Ponceau S. Incubate the membrane in Ponceau S stain for 1 to 15 min with gentle shaking/agitation. Rinse the membrane in MQ water until the background-signal ratio is good enough. Take a picture to confirm all the protein samples are equally transferred onto the membrane (Figure 3C). The stain can be completely removed from the protein bands by washing $5 \mathrm{~min}$ in $1 \mathrm{x}$ TBST.

Note: Incubation time in Ponceau S solution depends on the amount of protein in the membrane and of the strength of the reagent, the older is weaker. Ponceau $S$ is not recommended when the downstream application is analyzing phosphorylated proteins by Western blot because the alkaline conditions used in this step may modify phosphorylated residues.

14. Block the membrane with $15 \mathrm{ml}$ of blocking solution, shaking at $50 \mathrm{rpm}$ in a rocker at room temperature for $1 \mathrm{~h}$.

Note: Usually, blocking is done with $5 \%$ milk, but some antibodies need higher milk concentration to show a more specific signal. 6 to $7 \%$ milk may be used to increase specificity. When phosphorylated proteins will be detected, 2\% BSA is used for blocking instead of milk.

15. Wash the membrane in $1 x$ TBST, three times for 5 min each. All the washings and incubations are performed in a rocker at $50 \mathrm{rpm}$.

16. Incubate the membrane with the primary Antibody at 1:2,000 in 1x TBST, shaking over night at $4{ }^{\circ} \mathrm{C}$. For abundant proteins, $2 \mathrm{~h}$ at room temperature is a long enough incubation time.

Note: Primary and secondary antibody dilution must be empirically determined for each individual protein. Usually 1:2,000 of primary antibody and 1:3,000 of secondary antibody is enough for maize native proteins, but for very abundant proteins as the ones used as a loading control (e.g., Tubulin, Actin, Ubiquitin), or for recombinant proteins, dilutions as low as 1:20,000 are used.

17. Wash the membrane in $1 x$ TBST three times for 5 min each.

18. Incubate the membrane with the secondary Antibody at a dilution of $1: 3,000$ to $1: 10,000$ in $1 x$ TBST, shaking at room temperature for $2 \mathrm{~h}$. For abundant proteins, $1 \mathrm{~h}$ at room temperature is usually a long enough incubation time.

19. Wash the membrane in 1x TBST three times for 7-10 min each. Keep the membrane in the last washing buffer until ready to add the substrate, to prevent the membrane from drying.

20. To detect the protein, treat the membrane with peroxidase the ECL or NBT/BCIP substrate if the secondary antibody is conjugated with HRP (Horseradish Peroxidase) or AP (Alkaline phosphatase), respectively.

21. Place $800 \mu \mathrm{l}$ of the substrate on a plastic sheet (a piece of plastic wrap) and completely soak the membrane on both sides.

22. Incubate $5 \mathrm{~min}$ in the dark. Drain off excess substrate by taking the membrane with forceps and standing $10 \mathrm{~s}$ over two disposable wipes. Put the membrane between two transparent plastic layers preventing it from drying.

23. Place the wrapped membrane protein-side-up in an X-ray film cassette. In a dark room, expose to X-ray film for $30 \mathrm{~s}$ and develop it immediately. Depending on the signal intensity, adjust the 
exposure time until the desired signal-noise ratio is reached (Figure 4). Or use a Chemi-doc imager machine to capture the image. In this case, just place the wrapped membrane proteinside-up in the imager and use standard settings to capture, starting with $30 \mathrm{~s}$ exposure and increasing the time until the desired signal is reached.

24. If you wish to probe the membrane with a different primary antibody (e.g., Anti-Tubulin as a loading control, Figure 4), take out the membrane from the plastic wrapping and put it immediately in TBST buffer.

25. Wash the membrane in $1 x$ TBST for 5 min.

26. Add $15 \mathrm{ml}$ of restore Western blot stripping buffer to the membrane and shake it for $5 \mathrm{~min}$ at $50 \mathrm{rpm}$. Then wash it in $1 \times$ TBST for $5 \mathrm{~min}$.

27. Repeat Steps 12 to 19 using a different primary antibody and corresponding secondary antibody.

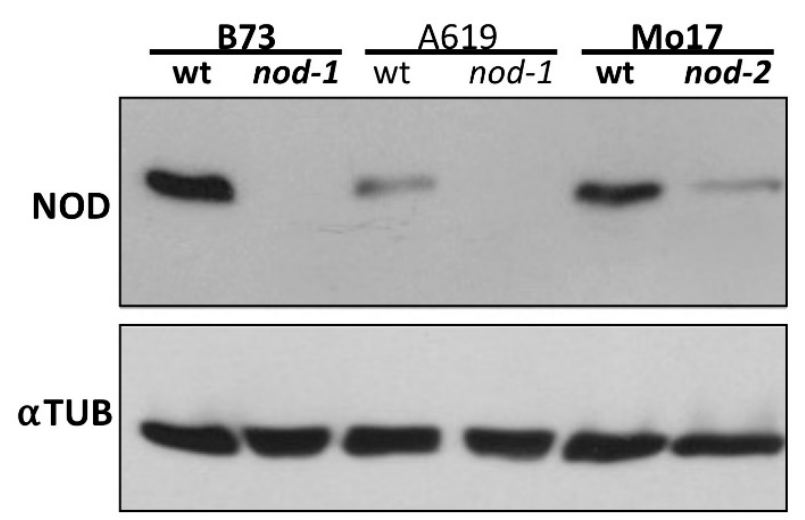

Figure 4. Western blot using a specific antibody Anti-NOD. Wild type and nod mutant shoot apical tissue from three different genetic backgrounds were used. As a loading control, an Antia Tubulin antibody was used. The primary antibody is a polyclonal Anti-NOD produced in Guinea pig (Rosa et al., 2017) and the secondary is a polyclonal Anti-Guinea pig HRPconjugated produced in Goat (Thermo Fisher Scientific).

\section{Data analysis}

1. Scan the developed X-ray films and save as JPEG format. Or save the Chemi-doc images as JPEG format.

2. Open the images using the ImageJ program and convert them to a gray-scale image by going to Image > Type > 8-bit.

3. Choose the "Rectangular Selections" tool from the toolbar and draw a rectangle around the first band (Figure 5A). Go to Analyze > Gels > Select First Lane to set the first band.

4. Click and hold in the middle of the rectangle on the first band and drag it over to the next band. Then go to Analyze $>$ Gels $>$ Select Next Lane to set the second band. Repeat Steps 3 and 4 until all the bands are selected, as shown in the NOD bands in Figure 5A. 
5. Go to Analyze $>$ Gels $>$ Plot Lanes to draw a profile plot for each lane (Figure 5B).

6. Choose the "Straight Line" selection tool from the tool-bar. For each band to analyze in the profile plot, draw a line across the base of the peak to enclose the peak.

7. Select the "Wand tracing" tool from the ImageJ toolbar, then click inside the peak. The area of the peak will be shown in a new window (Figure 5B, window). Record and save the peak area number for each band. Repeat this step until the peak areas of all the bands are collected.

8. Place the data in a spreadsheet (Figure $5 \mathrm{C}$ ). The first column is the peak areas of the interest protein detected using the primary antibody. The second column is the peak areas of the loading control protein (Tubulin) detected using the second antibody.

9. The relative density of the interest protein band is calculated by dividing the corresponding peak area by the loading control band from the same sample. The interest protein levels in the different samples can be compared, and the statistical analysis can be done using the relative density value (Gallo-Oller et al., 2018). Plots showing the relative intensity to the control protein can be done (Figure 5C).

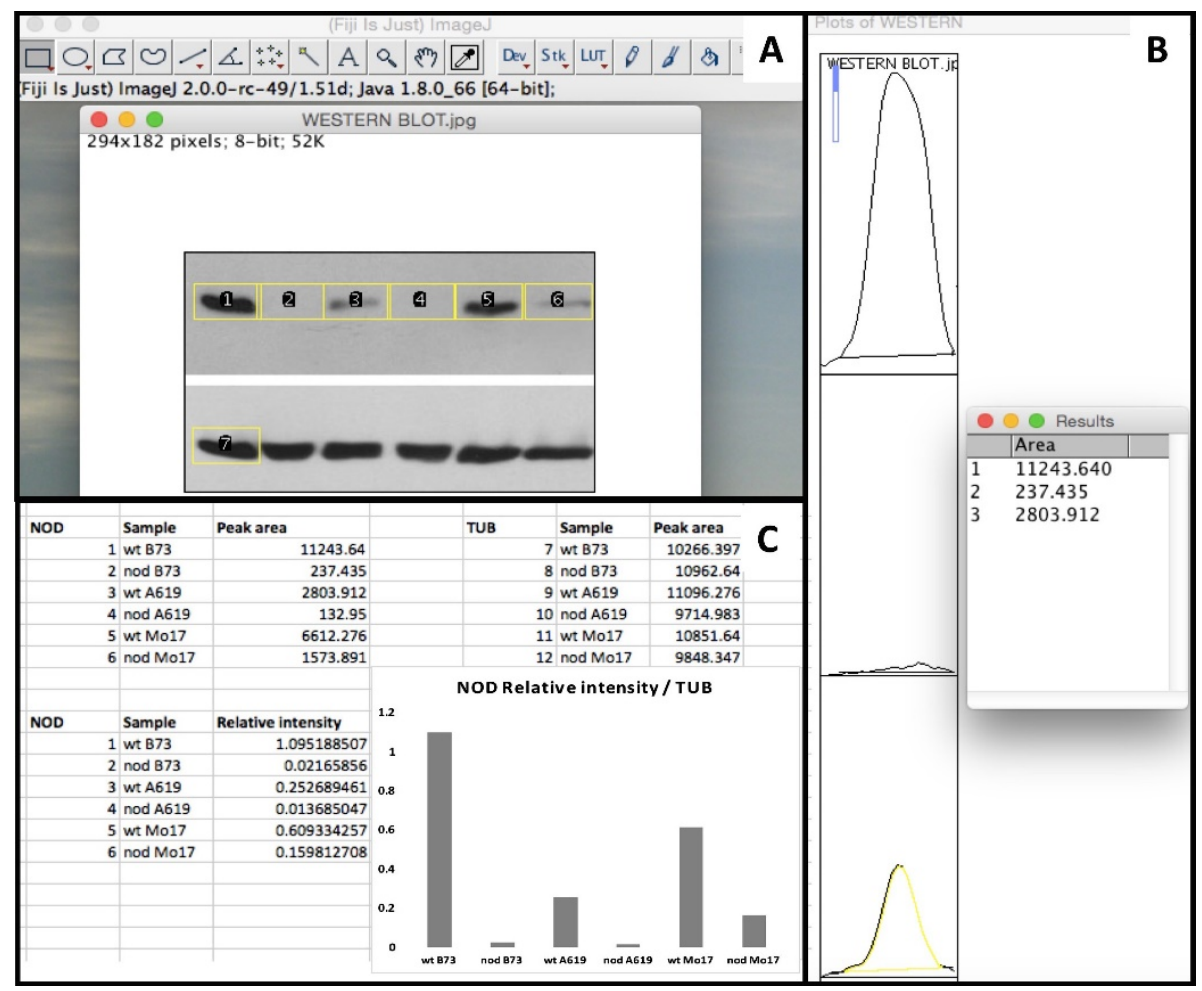

Figure 5. NOD Western blot data analysis using ImageJ. The protocol described here was used to get a graph showing the NOD relative intensity compared to the Tubulin control. A. ImageJ window showing rectangular sections in each band. B. Profile plots and peak areas for the first three NOD bands. C. Spreadsheet showing peak areas and relative intensity for the six samples, and the corresponding graph. This result is for a single replicate. To have Standard deviation and statistical analysis, at least three replicates are needed. 


\section{Notes}

1. Use freshly prepared $10 \%$ APS. It may be prepared and stored at $-20^{\circ} \mathrm{C}$ in small aliquots and used for one month. SDS-PAGE gels will not polymerize if old APS is used.

2. Chemiluminescent substrates differ in price and sensitivity. The ECL substrate described here, is used for standard protein concentration. If the protein expression is too low, it is advisable to use pico or femto level detection substrates. Also, when antibodies are very precious and higher dilutions are being used, femto level detection reagent is advisable.

\section{$\underline{\text { Recipes }}$}

1. Total protein extraction buffer (If using $100 \mathrm{mg}$ of tissue, only $200 \mu \mathrm{l}$ buffer will be needed for each sample. Prepare fresh buffer each time and store stock solutions at room temperature) $100 \mathrm{mM}$ Tris $\mathrm{pH} 7.2$

$10 \%$ sucrose

$5 \mathrm{mM} \mathrm{MgCl} 2$

5 mM EDTA

$40 \mu \mathrm{M} \beta$-mercaptoethanol

$2 x$ Protease inhibitor mix

1\% IGEPAL-CA-630

\section{$0.1 \%$ SDS}

Add PhosSTOPTM Phosphatase inhibitor mix (1 tablet for $10 \mathrm{ml}$ of extraction buffer) when phosphorylated proteins will be detected in the Western blot

2. $5 x$ Loading buffer (Prepare $5 \mathrm{ml}$ of buffer, make $0.5 \mathrm{ml}$ aliquots and store them at $-20{ }^{\circ} \mathrm{C}$ )

$0.25 \mathrm{M}$ Tris- $\mathrm{HCl}(\mathrm{pH} 6.8)$

$0.5 \mathrm{M}$ Dithiothreitol

$50 \%$ glycerol

$10 \%$ SDS

$0.25 \%$ bromophenol blue

3. $1 x$ Tris-Glycine running buffer $(900 \mathrm{ml}$ of the buffer is used for $2 \mathrm{gels}$ in a BioRad electrophoresis box. It may be reused until five times. Store at room temperature)

$25 \mathrm{mM}$ Tris- $\mathrm{HCl}$

$250 \mathrm{mM}$ glycine

$0.05 \%$ SDS

4. Transfer buffer (Prepare fresh mix each time, $100 \mathrm{ml}$ for each gel is used) $80 \% 1 x$ Tris Glycine buffer $20 \%$ Methanol

5. 1x TBST buffer (Aprox. $300 \mathrm{ml}$ are used for each western blot, store it at room temperature) $50 \mathrm{mM}$ Tris base 
$155 \mathrm{mM} \mathrm{NaCl}, \mathrm{pH} 7.6$

0.05\% Tween 20

6. Ammonium persulfate (APS)

$10 \%$ APS in MQ water (see Note 1)

7. Blocking solution (Prepare fresh each time, $20 \mathrm{ml}$ are used for each membrane) $5 \%$ nonfat milk (w/v) in $1 \times$ TBST buffer

8. Blocking solution for detection of phosphorylated proteins $2 \%$ BSA (w/v) in $1 \times$ TBST buffer

9. Ponceau S Staining Solution (Prepare fresh each time, $20 \mathrm{ml}$ is used for each membrane) $0.1 \%(w / v)$ Ponceau S in 5\% (v/v) acetic acid

\section{Acknowledgments}

MJAJ was supported by UC-MEXUS CONACYT and by NSF IOS-1238202 at Dr. Sarah Hake Lab (University of California Berkeley), where this protocol was developed. I would like to thank Dr. Sarah Hake and Dr. Madelaine Bartlett for their continuing scientific support, and to Dr. Ángel Gabriel Alpuche Solís and Alejandro Romo Avalos from IPICYT for their help in the preparation of figures and videos. I declare no conflict of interest regarding the implementation of this protocol.

\section{References}

1. Abraham-Juárez, M. J. (2019). Maize protein extraction for different downstream applications. Bio-101: e3241.

2. Gallo-Oller, G., Ordonez, R. and Dotor, J. (2018). A new background subtraction method for Western blot densitometry band quantification through image analysis software. $\mathrm{J}$ Immunol Methods 457: 1-5.

3. Rosa, M., Abraham-Juarez, M. J., Lewis, M. W., Fonseca, J. P., Tian, W., Ramirez, V., Luan, S., Pauly, M. and Hake, S. (2017). The maize MID-COMPLEMENTING ACTIVITY homolog CELL NUMBER REGULATOR13/NARROW ODD DWARF coordinates organ growth and tissue patterning. Plant Cell 29(3): 474-490.

4. Towbin, H., Staehelin, T. and Gordon, J. (1979). Electrophoretic transfer of proteins from polyacrylamide gels to nitrocellulose sheets: procedure and some applications. Proc Natl Acad Sci U S A 76(9): 4350-4354. 